\title{
BMJ Open Comparison of contemporary preoperative risk models at predicting acute kidney injury after isolated coronary artery bypass grafting: a retrospective cohort study
}

\author{
Shao-Wei Chen, ${ }^{1,2}$ Chih-Hsiang Chang, ${ }^{2,3}$ Pei-Chun Fan, ${ }^{2,3}$ Yung-Chang Chen, ${ }^{3}$ \\ Pao-Hsien Chu, ${ }^{4}$ Tien-Hsing Chen, ${ }^{4}$ Victor Chien-Chia Wu, ${ }^{4}$ Su-Wei Chang, ${ }^{5}$ \\ Pyng-Jing Lin, ${ }^{1}$ Feng-Chun Tsai ${ }^{1}$
}

To cite: Chen S-W, Chang C-H, Fan P-C, et al. Comparison of contemporary preoperative risk models at predicting acute kidney injury after isolated coronary artery bypass grafting:

a retrospective cohort study. BMJ Open 2016;6:e010176. doi:10.1136/bmjopen-2015010176

- Prepublication history and additional material is available. To view please visit the journal (http://dx.doi.org/ 10.1136/bmjopen-2015010176).

S-WC and C-HC contributed equally to this work.

Received 10 October 2015 Revised 8 May 2016 Accepted 6 June 2016

CrossMark

For numbered affiliations see end of article.

Correspondence to

Feng-Chun Tsai; 8702027@

cgmh.org.tw

\section{ABSTRACT}

Objectives: Acute kidney injury (AKI) after coronary artery bypass grafting $(\mathrm{CABG})$ is associated with shortterm and long-term adverse outcomes. The European System for Cardiac Operative Risk Evaluation (EuroSCORE), EuroSCORE II, the Society of Thoracic Surgeons (STS) score and Age, Creatinine and Ejection Fraction (ACEF) score, have been widely used for predicting the operative risk of cardiac surgery. The aim of this study is to investigate the discriminant ability among current available models in predicting postoperative AKI.

Methods: From January 2010 to December 2012, 353 patients who underwent isolated CABG were enrolled. The clinical characteristics, outcomes and scores of prognostic models were collected. The primary outcome was postoperative AKI, defined based on the Kidney Disease Improving Global Outcome (KDIGO) Clinical Practice Guideline for AKI, in 2012.

Results: 102 patients $(28.9 \%)$ developed postoperative AKI. For AKI prediction, EuroSCORE II, STS score and ACEF score were all good tools for stage-3 AKI. The ACEF score was shown to have satisfied discriminant ability to predict postoperative AKI with area under a receiver operating characteristic curve: $0.781 \pm 0.027,(95 \% \mathrm{Cl} 0.729$ to 0.834 , p value $<0.001)$. Multivariate logistic analysis identified that lower ejection fraction and higher serum creatinine were independent risk factors for AKI.

Conclusions: The simple and extremely user-friendly ACEF score can accurately identify the risk of postoperative AKI and has shown satisfactory discriminant ability when compared with other systems. The ACEF score might be the easiest tool for predicting postoperative AKI.

\section{INTRODUCTION}

Acute kidney injury (AKI) affects $12-48 \%$ of patients undergoing coronary artery bypass

\section{Strengths and limitations of this study}

- This is the first assessment comparing the prognostic utility of contemporary risk scoring systems for predicting post-operative acute kidney injury (AKI) in isolated coronary artery bypass grafting (CABG).

- We found that preoperative risk models in cardiac surgery, including European System for Cardiac Operative Risk Evaluation (EuroSCORE), EurOSCORE II, Society of Thoracic Surgeons score and Age, Creatinine and Ejection Fraction (ACEF score), can be used in predicting postoperative AKI in isolated CABG patients. The prediction ability was positively correlated with AKI severity.

- The simple and extremely user-friendly ACEF score can accurately identify the risk of postoperative AKI and has shown satisfactory discriminatory ability when compared with other systems; the ACEF score may be the easiest tool to provide guidance of preventive and early therapeutic strategies for AKI to improve the clinical outcomes of patients.

- Our study is limited by its post hoc analysis nature and all of its inherent limitations. Also, this series has the heterogeneity of off pump/on pump surgery, and urgent and elective surgery.

- Since the aetiology of AKI is often multifactorial, intraoperative factors and post-surgical care that were not involved in the scoring system may cause inaccurate prediction of AKI occurrence.

grafting $(\mathrm{CABG}) .{ }^{1}$ This statistical variant is dependent on different classifications and study populations. ${ }^{2-6}$ Postoperative AKI not only contributes to increased in-hospital mortality and decreased long-term survival, but also results in high medical expenditure, chronic kidney disease and dialysis dependence. $^{7-9}$ Since AKI has been known as a well- 
established predictor of all-cause mortality in CABG, an accurate and validated prediction model for AKI after cardiac surgery would be invaluable for clinical practices.

Numerous prognostic risk models for cardiac surgery have been introduced into the current practices. Among them, the European System for Cardiac Operative Risk Evaluation (EuroSCORE), published in $1999,{ }^{10}$ the revised EuroSCORE II, published in 2012, ${ }^{11}$ and the Society of Thoracic Surgeons (STS) score, published in 2008, have been widely used. ${ }^{12}$ The patient's age, creatinine and ejection fraction (ACEF) score was first described in 2009, in a publication for quick bedside evaluations. ${ }^{13}$ Although all these scoring models have been widely used for assessing the risk of post-surgical death, the various preoperative demographic and clinical variables have shown only that the STS database has successfully implicated the end point of renal failure. ${ }^{14}$

However, the benefits of utilising the risk model may be very limited due to the low dialysis rate in $1-5 \%$ cases. ${ }^{15}$ More effort is required to develop and validate different prediction scores to identify all levels of severity of AKI, since stage $1 \mathrm{AKI}$ is very common and contributes to short-term and long-term adverse outcomes with almost threefold increase in the long-term risk of end stage renal disease (ESRD), as demonstrated by a nationwide study. ${ }^{16}$ Nevertheless, no previous study has ever applied the contemporary preoperative risk modes to predict occurrence of AKI and its severity after surgery. The aim of this investigation is to compare the utility of scoring systems for predicting AKI after isolated CABG.

\section{PATIENTS AND METHODS}

\section{Study design and patient population}

This post hoc analysis of a prospective collected database was approved by the institution's research bureau (IRB) of Chang Gung Memorial Hospital, where the need for individual consent was waived. Medical records, from January 2010 to December 2012, of 440 consecutive patients who had received isolated CABG in a single tertiary referral hospital were reviewed. We excluded five patients who had had prior cardiac surgery, seven patients who had received extracorporeal membrane oxygenation (ECMO) immediately after surgery and six patients who had died on the day of surgery. In order to appraise renal outcomes, 57 patients who had undergone dialysis and 12 patients who had experienced AKI before their operation, were also excluded. The final cohort comprised a total of 353 patients.

\section{Data collection and definitions}

Clinical characteristics, demographic data and EuroSCORE I were extracted from the database. The STS score and EuroSCORE II were re-calculated with an online calculator. The ACEF score was calculated by age/ejection fraction $(\mathrm{EF})+1$ (if creatinine $>2.0 \mathrm{mg}$ / $\mathrm{dL}$ ). The primary outcome was AKI and 3-month mortality. Base on the KDIGO Clinical Practice Guideline for Acute Kidney Injury, AKI was defined as any of the following: increase in serum creatinine $(\mathrm{SCr}) \geq 0.3 \mathrm{mg} / \mathrm{dL}$ within 48 hours or increase in $\mathrm{SCr} \geq 1.5$ times baseline in 7 days or urine volume $<0.5 \mathrm{~mL} / \mathrm{kg} /$ hour for 6 hours. Urine output and SCr level were routinely measured and the data collected in our medical records. Finally, patients were categorised into three different severities, using the KDIGO guideline ${ }^{17} 18$ (table 1).

\section{Statistical analysis}

Continuous variables were summarised as mean and $\mathrm{SE}$ unless otherwise stated. The primary end point was the comparison between AKI and non-AKI groups. The Kolmogorov-Smirnov test was used to determine the normal distribution for each variable, and the Student's t-test was used to compare the means of continuous variables and normally distributed data; otherwise, the Mann-Whitney U test was used. Categorical data were

Table 1 Staging and definition of AKI according to the KDIGO guidelines in 2012

\begin{tabular}{|c|c|c|c|}
\hline Definition & Stage & Serum creatinine & Urine output \\
\hline $\begin{array}{l}\text { Increase in } \mathrm{SCr} \text { by } \geq 0.3 \mathrm{mg} / \mathrm{dL} \\
\text { ( } \geq 26.5 \mu \mathrm{mol} / \mathrm{L}) \text { within } 48 \text { hours } \\
\text { or } \\
\text { Increase in } \mathrm{SCr} \text { to } \geq 1.5 \text { times } \\
\text { baseline, which is known or } \\
\text { presumed to have occurred } \\
\text { within the prior } 7 \text { days } \\
\text { or } \\
\text { Urine volume }<0.5 \mathrm{~mL} / \mathrm{kg} / \mathrm{hour} \\
\text { for } 6 \text { hours }\end{array}$ & $\begin{array}{l}2 \\
3\end{array}$ & $\begin{array}{l}1.5-1.9 \text { times baseline } \\
\text { or } \\
\geq 0.3 \mathrm{mg} / \mathrm{dL}(\geq 26.5 \mu \mathrm{mol} / \mathrm{L}) \text { increase } \\
2.0-2.9 \text { times baseline } \\
3.0 \text { times baseline } \\
\text { or } \\
\text { Increase in serum creatinine to } \geq 4.0 \mathrm{mg} / \mathrm{dL} \\
\text { ( } \geq 353.6 \mu \mathrm{mol} / \mathrm{L}) \\
\text { or } \\
\text { Initiation of renal replacement therapy } \\
\text { or, } \\
\text { In patients }<18 \text { years, decrease in eGFR to } \\
<35 \mathrm{~mL} / \mathrm{min} \text { per } 1.73 \mathrm{~m}^{2}\end{array}$ & $\begin{array}{l}<0.5 \mathrm{~mL} / \mathrm{kg} / \text { hour for } \geq 12 \text { hour } \\
<0.3 \mathrm{~mL} / \mathrm{kg} / \text { hour for } \geq 24 \text { hours } \\
\text { or } \\
\text { Anuria for } \geq 12 \text { hour }\end{array}$ \\
\hline
\end{tabular}


tested using the $\chi^{2}$ test or Fisher's exact test. Risk factors for AKI were assessed using univariate analysis, and variables that were statistically significant in the univariate analysis were included in multivariate logistic regression analysis based on the backward elimination of data. Discrimination was assessed using area under a receiver operating characteristic curve (AUROC) analysis, and the discriminatory ability between the ACEF and other scoring systems was compared using a non-parametric approach. The AUROC analysis calculated cut-off values, sensitivity, specificity and overall correctness. Finally, cut-off points were calculated using the best Youden index, defined as sensitivity+specificity-1, where sensitivity and specificity were calculated as proportions. Youden's index has minimum and maximum values of -1 and +1 , respectively, with a value of +1 representing the optimal value for an algorithm. A $\mathrm{p}$ value of $<0.05$ was considered to indicate statistical significance.

\section{RESULTS}

Characteristics of the study population: non-AKI versus AKI groups

The study cohort consisted of 353 adult patients with a mean age of $64 \pm 1.0$ years $(288(81.6 \%)$ males and 65 females), of whom 102 (28.9\%) developed postoperative AKI (46 (45.1\%) stage I, $14(13.7 \%)$ stage II and 42 (41.2\%) stage III). All clinical characteristics are listed in table 2. Compared to the patients without AKI, those with AKI were older, had a higher frequency of diabetes mellitus (DM), higher level of SCr, lower level of albumin and poorer preoperative heart condition including lower EF, higher frequency of the use of preoperative intra-aortic

Table 2 Demographic data and clinical characteristics of patients with/without AKI during their hospital course (expression as mean $\pm \mathrm{SE}$ )

\begin{tabular}{|c|c|c|c|c|}
\hline & All Patients $(n=353)$ & Non-AKI $(n=251)$ & AKI $(n=102)$ & p Value \\
\hline \multicolumn{5}{|l|}{ Preoperative demographic data } \\
\hline Age (years) & $64 \pm 1$ & $63 \pm 1$ & $67 \pm 1$ & 0.001 \\
\hline Gender, male (\%) & $288(81.6)$ & $202(80.5)$ & $86(84.3)$ & 0.399 \\
\hline Diabetes mellitus (\%) & $176(49.9)$ & $112(44.6)$ & $64(62.7)$ & 0.002 \\
\hline Hypertension (\%) & $275(77.9)$ & $186(74.1)$ & 89 (87.3) & 0.007 \\
\hline ALT (units/L) & $36 \pm 3$ & $32 \pm 2$ & $47 \pm 12$ & 0.220 \\
\hline Serum creatinine (mg/dL) & $1.1 \pm 0.0$ & $1.0 \pm 0.0$ & $1.5 \pm 0.1$ & $<0.001$ \\
\hline Albumin $(g / L)$ & $3.9 \pm 0.0$ & $4.0 \pm 0.0$ & $3.7 \pm 0.1$ & $<0.001$ \\
\hline Haemoglobin (g/dL) & $12.9 \pm 0.1$ & $13.1 \pm 0$ & $12.3 \pm 0.2$ & 0.006 \\
\hline hs-CRP (mg/L) & $20.0 \pm 2.4$ & $15.3 \pm 2.2$ & $31.5 \pm 5.7$ & $<0.001$ \\
\hline \multicolumn{5}{|l|}{ Preoperative heart condition } \\
\hline CAD vessels & $2.8 \pm 0.1$ & $2.8 \pm 0.1$ & $2.9 \pm 0.1$ & 0.049 \\
\hline Recent MI, (\%) & $157(44.5)$ & $96(38.2)$ & $61(59.8)$ & $<0.001$ \\
\hline CHF Fc III/IV (\%) & $51(14.4)$ & $22(8.8)$ & $29(28.4)$ & $<0.001$ \\
\hline Ejection fraction (\%) & $54 \pm 1$ & $57 \pm 2$ & $45 \pm 6$ & $<0.001$ \\
\hline Inotropic agent (\%) & $38(10.8)$ & $21(8.4)$ & $17(16.7)$ & 0.023 \\
\hline Mechanical ventilation, $\mathrm{n}(\%)$ & $29(8.2)$ & $11(4.4)$ & $18(17.6)$ & $<0.001$ \\
\hline $\mathrm{IABP},(\%)$ & $37(10.5)$ & $14(5.6)$ & $23(22.5)$ & $<0.001$ \\
\hline \multicolumn{5}{|l|}{ Preoperative scores } \\
\hline STS-risk of mortality & $5.7 \pm 0.6$ & $3.4 \pm 0.4$ & $11.2 \pm 1.8$ & $<0.001$ \\
\hline STS-renal failure & $6.9 \pm 0.7$ & $3.7 \pm 0.4$ & $14.9 \pm 1.7$ & $<0.001$ \\
\hline EuroSCORE I & $12.6 \pm 0.9$ & $8.7 \pm 0.8$ & $22.2 \pm 2.4$ & $<0.001$ \\
\hline EuroSCORE II & $5.4 \pm 0.5$ & $3.5 \pm 0.3$ & $10.3 \pm 1.3$ & $<0.001$ \\
\hline ACEF & $1.4 \pm 0.1$ & $1.2 \pm 0.1$ & $1.9 \pm 0.1$ & $<0.001$ \\
\hline \multicolumn{5}{|l|}{ Surgical detail } \\
\hline Urgent operation (\%) & $87(24.6)$ & $48(19.1)$ & $39(38.2)$ & $<0.001$ \\
\hline On pump CABG (\%) & $243(68.8)$ & $158(62.9)$ & 85 (83.3) & $<0.001$ \\
\hline Aortic clamp time (minutes) & $91.3 \pm 3.4$ & $87.0 \pm 3.2$ & $96.4 \pm 6.6$ & 0.184 \\
\hline Cardiopulmonary bypass time (minutes) & $117.3 \pm 2.9$ & $109.6 \pm 2.8$ & $131.4 \pm 6.2$ & 0.002 \\
\hline Bypass graft number & $3.0 \pm 0.1$ & $3.0 \pm 0$ & $3.1 \pm 0$ & 0.167 \\
\hline \multicolumn{5}{|l|}{ Patient outcome } \\
\hline ICU stay (days) & $5.3 \pm 0.8$ & $2.9 \pm 0.2$ & $7.9 \pm 1.1$ & $<0.001$ \\
\hline Ventilator duration (hours) & $38.3 \pm 7.5$ & $22.8 \pm 2.6$ & $58.4 \pm 11.8$ & 0.004 \\
\hline AKI stage $1 / 2 / 3$ & - & - & $46 / 14 / 42$ & - \\
\hline Renal replacement therapy $n(\%)$ & $28(7.9)$ & - & $28(27.4)$ & - \\
\hline 30-day mortality & $20(5.7)$ & $1(0.4)$ & $19(18.6)$ & $<0.001$ \\
\hline
\end{tabular}

ACEF, age, creatinine and ejection fraction; AKI, acute kidney injury; ALT, alanine transaminase; CAD, coronary artery disease; CHF Fc, congestive heart failure functional class; hs-CRP, high-sensitivity $C$ reactive protein; IABP, intra-aortic balloon pump; ICU, intensive care unit; MI, myocardial infarction; NS, not significant; STS, Society of Thoracic Surgeons. 
Table 3 Comparison of calibration and discrimination of the scoring system in predicting AKIs of differing severity

\begin{tabular}{|c|c|c|c|c|c|c|}
\hline \multirow[b]{2}{*}{ Scoring system } & \multicolumn{3}{|l|}{ All AKI } & \multicolumn{3}{|l|}{ AKI stage 3} \\
\hline & $\overline{\mathrm{AUROC} \pm \mathrm{SE}}$ & $95 \% \mathrm{Cl}$ & p Value & $\overline{A U R O C} \pm S E$ & $95 \% \mathrm{Cl}$ & p Value \\
\hline STS-risk of mortality & $0.714 \pm 0.031$ & 0.653 to 0.775 & $<0.001$ & $0.843 \pm 0.032$ & 0.781 to 0.906 & $<0.001$ \\
\hline STS-renal failure & $0.765 \pm 0.029$ & 0.709 to 0.822 & $<0.001$ & $0.892 \pm 0.025$ & 0.842 to 0.942 & $<0.001$ \\
\hline EuroSCORE I & $0.697 \pm 0.032$ & 0.635 to 0.759 & $<0.001$ & $0.776 \pm 0.038$ & 0.701 to 0.851 & $<0.001$ \\
\hline EuroSCORE II & $0.738 \pm 0.030$ & 0.680 to 0.797 & $<0.001$ & $0.851 \pm 0.030$ & 0.793 to 0.910 & $<0.001$ \\
\hline ACEF & $0.781 \pm 0.027$ & 0.729 to 0.834 & $<0.001$ & $0.838 \pm 0.034$ & 0.772 to 0.904 & $<0.001$ \\
\hline
\end{tabular}

ACEF, age, creatinine and ejection fraction; AKI, acute kidney injury; AUROC, areas under the receiver operating characteristic curve; STS, Society of Thoracic Surgeons.

balloon pumping (IABP), more recent myocardial infarction (MI) and more vessels with coronary artery disease. The mean EuroSCORE I, EuroSCORE II and STS scores for the risk of mortality were $12.6 \pm 0.9 \%, 5.4 \pm 0.5 \%$ and $5.7 \pm 0.6 \%$, respectively. All preoperative scores were significantly different between the AKI and non-AKI groups. Twenty (5.7\%) patients died within 30 days, with a significantly higher rate in the AKI group than in the non-AKI group $(18.6 \%$ vs $0.4 \% ; \mathrm{p}<0.001)$. Furthermore, the patients in the AKI group also had longer durations of ventilator use and intensive care unit stay.

\section{Scoring systems and AKI prediction}

We tested the four mortality scores for their discriminatory ability in predicting all AKI and stage 3 AKI (table 3). The ACEF score had the best AUROC (0.781 \pm 0.027 ) to predict postoperative AKI among the five scoring systems, followed by STS-renal failure $(0.765$ $\pm 0.029)$, EuroSCORE II $(0.738 \pm 0.030)$, STS-risk of mortality $(0.714 \pm 0.031)$ and EuroSCORE I $(0.697 \pm 0.032)$. The ACEF score outperformed the STS-risk of mortality $(p=0.016)$ and EuroSCORE I $(p=0.003)$, but it was comparable with STS-renal failure and EuroSCORE II (see online supplementary table S1). In subgroup analysis, all the scoring systems, including EuroSCORE, had significant discriminatory ability for AKI stage 3 . The AUROC was highest for STS-renal failure $(0.892 \pm 0.025)$, followed by EuroSCORE II $(0.851 \pm 0.030)$, STS-risk of mortality $(0.843 \pm 0.032)$ and ACEF $(0.838 \pm 0.034)$, and lowest for EuroSCORE I $(0.776 \pm 0.038)$. The performance between the ACEF score and the other four scoring systems was comparable (see online supplementary table S1).

In order to assess cut-off points to predict AKI, we determined the sensitivity and specificity (table 4 ). The optimal cut-off point for the ACEF score to predict AKI was 1.2 , with a sensitivity of $82 \%$ and specificity of $67 \%$. With regard to predicting stage 3 AKI, a cut-off ACEF value of 1.5 had an even higher sensitivity of $83 \%$, and specificity of $72 \%$.

To determine the discriminatory ability of the STS-renal failure score, we plotted calibration curves by quintile of STS-renal failure score for all AKIs (figure 1). This score was found to underestimate postoperative AKI in all quintiles, with increasing discrepancy in the higher quintiles.

\section{Logistic regression analysis for AKI according to preoperative variable}

A logistic regression model was used for the patients with AKI according to the preoperative factors. After incorporating the significant independent variables in the univariate analysis into the multiple logistic regression analysis with backward selection, only lower ejection fraction and higher SCr level were independently associated with the risk of postoperative AKI (table 5).

Table 4 Prediction of AKI in different scoring systems in isolated coronary artery bypass surgery

\begin{tabular}{lllll}
\hline Predictive factors & Cut-off point & Youden index & Sensitivity (\%) & Specificity (\%) \\
\hline All AKI & & & & \\
STS-risk of mortality & 2.3 & 0.35 & 61 & 75 \\
STS-renal failure & 4.9 & 0.42 & 60 & 82 \\
EuroSCORE I & 5.9 & 0.35 & 72 & 63 \\
EuroSCORE II & 3.0 & 0.39 & 70 & 68 \\
ACEF & 1.2 & 0.46 & 82 & 67 \\
AKI stage 3 & & & 80 \\
STS-risk of mortality & 3.9 & 0.56 & 76 & 81 \\
STS-renal failure & 5.9 & 0.65 & 74 & 67 \\
EuroSCORE I & 9.1 & 0.41 & 88 & 64 \\
EuroSCORE II & 3.2 & 0.52 & 83 & 72 \\
ACEF & 1.5 & 0.55 &
\end{tabular}




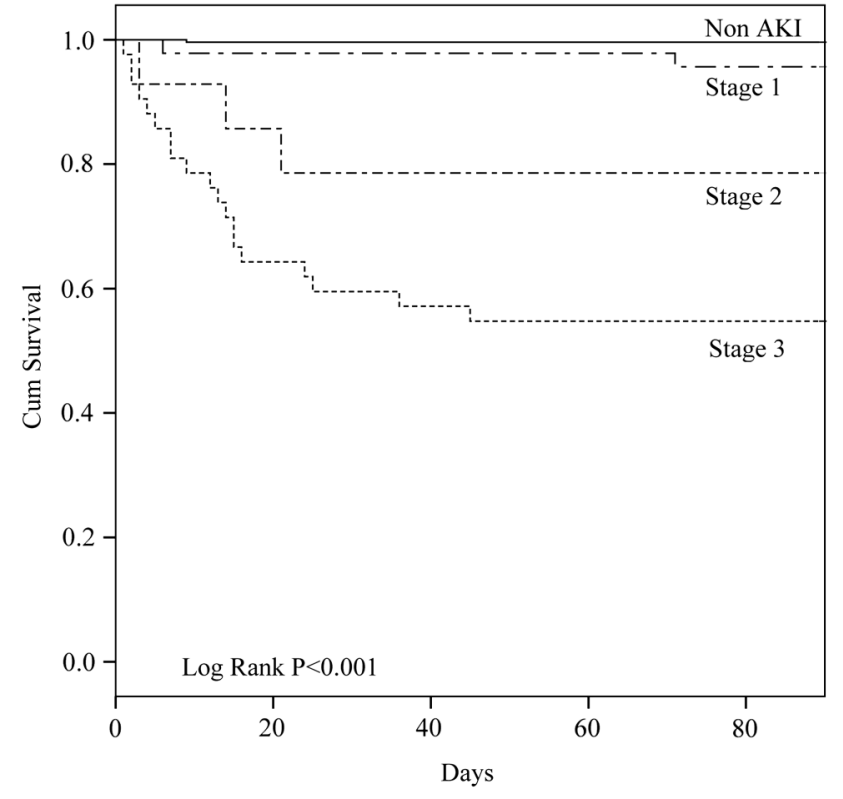

Figure 1 Quintile calibration plots of STS-renal failure for expected $\mathrm{AKI}$ and observed $\mathrm{AKI}$. AKI, acute kidney injury; STS, Society of Thoracic Surgeons.

\section{Short-term outcomes}

Figure 2 illustrates the cumulative survival rate of all 353 isolated CABG patients stratified by the severity of AKI. As expected, the patients with stage 2 or 3 AKI had a significantly lower survival rate $(\log \operatorname{rank} \mathrm{p}<0.001)$. Compared to the patients without AKI, although there was no significant difference in survival, those with stage 1 AKI had a higher number of admissions for recurrent angina and hyperkalaemia during follow-up.

\section{DISCUSSION}

The development of AKI is associated with unfavourable outcomes and a high mortality rate in patients undergoing isolated CABG. The mechanisms of AKI after cardiac surgery are multifactorial, and can include ischaemic-reperfusion, cytokine release, haemolysis, oxidase stress and exposure to nephrotoxins. All of these factors can result in endothelial dysfunction, inflammatory responses and tubule cell damage. AKI after cardiac surgery can be classified as a form of cardiorenal syndrome type I, a bidirectional condition that reflects an abrupt degradation of renal function, secondary to acute cardiac disease or procedures. ${ }^{19}$ Since renal dysfunction is a well-established predictor of all-cause mortality in patients undergoing CABG, an accurate and validated prediction model for AKI after cardiac surgery would be invaluable to facilitate clinical decisionmaking, patient counselling and preoperative medical optimisation. $^{20}$ Currently, the prediction models for severe AKI requiring dialysis are the most robust and externally validated. Among them, the STS score for renal failure has been shown to have a high discriminatory ability in most tested populations. ${ }^{21}$ However, due to the low dialysis rate $(1-2 \%)$ and late occurrence, the benefits of using these risk models may be limited, and further research is required to develop and validate different prediction scores to identify all severities of AKI, since stage $1 \mathrm{AKI}$ is very common, and contributes to many short-term and long-term adverse outcomes.

Several validated scoring models, including EuroSCORE I and II, and STS score, have been developed and are widely used to assess the risk of mortality in patients undergoing cardiac surgery. These risk scores are based on collected data and use online tools to calculate scores, which can then predict the risk of mortality from cardiac surgery based on patient demographics and clinical variables. Recently, these scoring systems have been expanded to incorporate clinical characteristics specific for cardiac surgery, and have been applied to patients undergoing percutaneous coronary interventions due to the similar study population. ${ }^{22-24}$ Thus, these contemporary preoperative mortality risk scoring systems have been used to predict major complications such as postoperative AKI. Furthermore, our recent study demonstrated that the STS score is also an effective tool to predict AKI in patients with coronary artery disease without CABG. ${ }^{25}$ In another study, Ando et al ${ }^{26}$ demonstrated that the ACEF score can be used as a predictor of AKI in patients undergoing primary percutaneous coronary interventions. The major risk factors for postoperative mortality after cardiac surgery are well known, and postoperative major complications share the same risk factors that may contribute to the development of AKI, such as decreased left ventricle ejection fraction and increased SCr level, as demonstrated in this study. We also demonstrated that the current mortality risk scoring systems can be used to predict post-CABG AKI.

The ACEF score is based on the 'law of parsimony' to exclude some of the confounders that could bias the other current scoring systems, where the incorporation of too many variables has resulted in inaccuracies and poor overfitting. ${ }^{13}$ Further, Andò et $a l^{27}$ demonstrated that the predictive ability of the ACEF score can, often, be refined by the use of additional prognostic indicators that are specific to the population studied. A recent study reported that the ACEF score is a similar or more accurate model to stratify the risk of mortality in patients undergoing $\mathrm{CABG}$ and in patients undergoing percutaneous coronary interventions. ${ }^{28}$ This simple score combines three important clinical variables: age, creatinine (renal insufficiency) and left ventricular ejection fraction. These three preoperative clinical variables are well known independent risk factors for postoperative AKI in patients undergoing cardiac surgery. Therefore, the ACEF scoring system may be a useful and applicable risk model to predict postoperative AKI. Furthermore, because it uses clinical variables that can be obtained easily and quickly, it is more suitable for non-elective surgery. A recent study demonstrated that the performance of the ACEF score in predicting in-hospital mortality in elective and non-elective cardiac surgery is 
Table 5 Logistic regression analysis of presurgical factors for all AKI

\begin{tabular}{|c|c|c|c|c|}
\hline Parameter & $\beta$-Coefficient & SE & OR $(95 \% \mathrm{Cl})$ & p Value \\
\hline \multicolumn{5}{|c|}{ Univariate logistic regression } \\
\hline Age & 0.036 & 0.011 & $1.037(1.015$ to 1.060$)$ & 0.001 \\
\hline Diabetes mellitus & 0.737 & 0.241 & 2.090 (1.303 to 3.352$)$ & 0.002 \\
\hline Hypertension & -0.872 & 0.330 & $0.418(0.219$ to 0.798$)$ & 0.008 \\
\hline Inotropic agent & 0.784 & 0.350 & $2.190(1.103$ to 4.350$)$ & 0.025 \\
\hline Mechanical ventilation & -1.542 & 0.403 & 0.214 (0.097 to 0.471 ) & $<0.001$ \\
\hline Serum creatinine & 2.114 & 0.329 & 8.284 (4.346 to 15.792$)$ & $<0.001$ \\
\hline Albumin & -1.349 & 0.354 & $0.260(0.130$ to 0.520$)$ & $<0.001$ \\
\hline Haemoglobin & -0.146 & 0.054 & 0.864 (0.777 to 0.961$)$ & 0.007 \\
\hline hs-CRP & 0.016 & 0.005 & $1.016(1.005$ to 1.027$)$ & 0.004 \\
\hline IABP & -1.595 & 0.363 & $0.203(0.100$ to 0.413$)$ & 0.001 \\
\hline Ejection fraction & -0.048 & 0.008 & 0.953 (0.938 to 0.968$)$ & $<0.001$ \\
\hline Recent MI & 0.876 & 0.240 & $2.402(1.500$ to 3.846$)$ & $<0.001$ \\
\hline CAD vessels & 0.511 & 0.296 & $1.667(0.993$ to 2.980$)$ & 0.084 \\
\hline \multicolumn{5}{|c|}{ Multivariate logistic regression } \\
\hline Age & 0.034 & 0.019 & 1.035 (0.997 to 1.074$)$ & 0.072 \\
\hline Serum creatinine & 3.005 & 0.667 & 20.283 (5.459 to 70.659$)$ & $<0.001$ \\
\hline Ejection fraction & -0.042 & 0.014 & 0.959 (0.933 to 0.986$)$ & 0.003 \\
\hline CHF Fc III/IV & 0.934 & 0.537 & 2.545 (0.889 to 7.287 ) & 0.082 \\
\hline constant & -4.421 & 1.385 & - & - \\
\hline
\end{tabular}

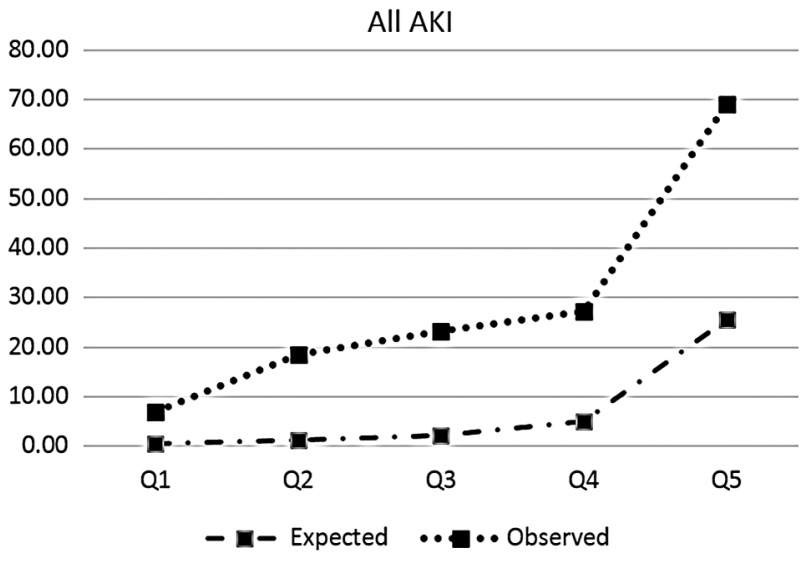

Figure 2 Short-term survival according to non-AKI and AKI groups. AKI, acute kidney injury.

comparable. ${ }^{29}$ We also demonstrated its superior application in predicating all severities of AKI in elective and non-elective surgery. Currently, novel biomarkers allow for the early detection of postoperative AKI, however, the cost of screening every case may be a concern. ${ }^{30} 31$ Hence, combining risk assessment tools and postsurgical biomarkers may improve the cost efficacy in daily practice.

\section{Study limitations}

There are several limitations to this study. First, the nature of post hoc analysis and all of its inherent limitations. Second, this series has a relatively small number of cases, with heterogeneity for off pump/on pump surgery, and urgent and elective surgery. Finally, this study was conducted at a single tertiary care medical centre in Taiwan, and thus the results may not be directly extrapolated to other patient populations. Finally, since the aetiology of AKI is often multifactorial, scoring systems not including intraoperative factors and postsurgical care may cause inaccuracies in the prediction of the risk of AKI.

\section{CONCLUSIONS}

To the best of our knowledge, this is the first study to compare the prognostic use of contemporary risk scoring systems to predict postoperative AKI in patients undergoing isolated CABG. The principle findings of the current study are: first, preoperative risk models, including the EuroSCORE II, STS and ACEF scoring systems, can be used to predict postoperative AKI in patients undergoing isolated CABG. The prediction ability was positively correlated with the severity of AKI. Second, the multivariate analysis identified a low ejection fraction and high SCr level to be independently associated with postoperative AKI. Third, the simple ACEF score can accurately identify the risk of postoperative AKI with satisfactory discriminatory ability compared with the other systems. In conclusion, the ACEF score may be the best and easiest tool to guide preventive and early therapeutic strategies for AKI to improve patient clinical outcomes. Future studies should be focused on combining preoperative risk models and postoperative AKI biomarkers to allow for an accurate diagnosis for future clinical management. 
Author affiliations

${ }^{1}$ Department of Cardiothoracic and Vascular Surgery, Chang Gung Memorial Hospital, Linkou Branch, Taoyuan, Taiwan

${ }^{2}$ Graduate Institute of Clinical Medical Sciences, College of Medicine, Chang Gung University, Taoyuan, Taiwan

${ }^{3}$ Department of Nephrology, Kidney Research Center, Chang Gung Memorial Hospital, Linkou Branch, Taoyuan, Taiwan

${ }^{4}$ Department of Cardiology, Chang Gung Memorial Hospital, Linkou Branch, Taoyuan, Taiwan

${ }^{5}$ Clinical Informatics and Medical Statistics Research Center, Taoyuan, Taiwan

Contributors S-WC and F-CT are consultant cardiac surgeons. C-HC created and designed this study. S-WC analysed the data with P-CF and Y-CC. P-HC, T-HC, VC-CW and P-JL contributed to the preparation and editing of the manuscript.

Funding This research received no specific grant from any funding agency in the public, commercial or not-for-profit sectors.

Competing interests None declared.

Ethics approval Institution's research bureau (IRB) of Chang Gung Memorial Hospital.

Provenance and peer review Not commissioned; externally peer reviewed.

Data sharing statement No additional data are available.

Open Access This is an Open Access article distributed in accordance with the Creative Commons Attribution Non Commercial (CC BY-NC 4.0) license, which permits others to distribute, remix, adapt, build upon this work noncommercially, and license their derivative works on different terms, provided the original work is properly cited and the use is non-commercial. See: http:// creativecommons.org/licenses/by-nc/4.0/

\section{REFERENCES}

1. Oezkur M, Wagner M, Weismann $\mathrm{D}$, et al. Chronic hyperglycemia is associated with acute kidney injury in patients undergoing CABG surgery-a cohort study. BMC Cardiovasc Disord 2015;15:41.

2. Gallagher S, Jones DA, Lovell MJ, et al. The impact of acute kidney injury on midterm outcomes after coronary artery bypass graft surgery: a matched propensity score analysis. $J$ Thorac Cardiovasc Surg 2014;147:989-95.

3. Ryden L, Ahnve S, Bell M, et al. Acute kidney injury after coronary artery bypass grafting and long-term risk of myocardial infarction and death. Int J Cardiol 2014;172:190-5.

4. Rosner MH, Okusa MD. Acute kidney injury associated with cardiac surgery. Clin J Am Soc Nephrol 2006;1:19-32.

5. Chen YC, Tsai FC, Chang CH, et al. Prognosis of patients on extracorporeal membrane oxygenation: the impact of acute kidney injury on mortality. Ann Thorac Surg 2011;91:137-42.

6. Oezkur M, Gorski A, Peltz J, et al. Preoperative serum h-FABP concentration is associated with postoperative incidence of acute kidney injury in patients undergoing cardiac surgery. BMC Cardiovasc Disord 2014;14:117.

7. Parikh CR, Coca SG, Wang Y, et al. Long-term prognosis of acute kidney injury after acute myocardial infarction. Arch Intern Med 2008;168:987-95.

8. Chen $\mathrm{TH}$, Chang $\mathrm{CH}$, Lin $\mathrm{CY}$, et al. Acute kidney injury biomarkers for patients in a coronary care unit: a prospective cohort study. PLOS ONE 2012;7:e32328.

9. Lin CY, Tsai FC, Tian YC, et al. Evaluation of outcome scoring systems for patients on extracorporeal membrane oxygenation. Ann Thorac Surg 2007;84:1256-62.
10. Nashef SA, Roques F, Michel $\mathrm{P}$, et al. European system for cardiac operative risk evaluation (EuroSCORE). Eur J Cardiothorac Surg 1999;16:9-13.

11. Nashef SA, Roques F, Sharples LD, et al. EuroSCORE II. Eur J Cardiothorac Surg 2012;41:734-44; discussion 44-5.

12. O'Brien SM, Shahian DM, Filardo G, et al. The Society of Thoracic Surgeons 2008 cardiac surgery risk models: part 2-isolated valve surgery. Ann Thorac Surg 2009;88(1 Suppl):S23-42.

13. Ranucci M, Castelvecchio S, Menicanti L, et al. Risk of assessing mortality risk in elective cardiac operations: age, creatinine, ejection fraction, and the law of parsimony. Circulation 2009;119:3053-61.

14. Mehta RH, Grab JD, O'Brien SM, et al. Bedside tool for predicting the risk of postoperative dialysis in patients undergoing cardiac surgery. Circulation 2006;114:2208-16; quiz 08.

15. Chertow GM, Lazarus JM, Christiansen CL, et al. Preoperative renal risk stratification. Circulation 1997;95:878-84.

16. Ryden L, Sartipy U, Evans M, et al. Acute kidney injury after coronary artery bypass grafting and long-term risk of end-stage renal disease. Circulation 2014;130:2005-11.

17. Palevsky PM, Liu KD, Brophy PD, et al. KDOQI US commentary on the $2012 \mathrm{KDIGO}$ clinical practice guideline for acute kidney injury. Am J Kidney Dis 2013;61:649-72.

18. Okusa MD, Davenport A. Reading between the (guide)lines-the KDIGO practice guideline on acute kidney injury in the individual patient. Kidney Int 2014:85:39-48.

19. Ronco C, Haapio M, House AA, et al. Cardiorenal syndrome. J Am Coll Cardiol 2008;52:1527-39.

20. Thakar CV. Predicting acute kidney injury after cardiac surgery: how to use the "crystal ball". Am J Kidney Dis 2010;56:605-8.

21. Huen SC, Parikh CR. Predicting acute kidney injury after cardiac surgery: a systematic review. Ann Thorac Surg 2012;93:337-47.

22. Kim YH, Ahn JM, Park DW, et al. EuroSCORE as a predictor of death and myocardial infarction after unprotected left main coronary stenting. Am J Cardiol 2006;98:1567-70.

23. Romagnoli E, Burzotta F, Trani C, et al. EuroSCORE as predictor of in-hospital mortality after percutaneous coronary intervention. Heart 2009;95:43-8.

24. Rodes-Cabau J, Deblois J, Bertrand OF, et al. Nonrandomized comparison of coronary artery bypass surgery and percutaneous coronary intervention for the treatment of unprotected left main coronary artery disease in octogenarians. Circulation 2008;118:2374-81.

25. Chang $\mathrm{CH}$, Fu CM, Yang $\mathrm{CH}$, et al. Society of Thoracic Surgeons Score predicts kidney injury in patients not undergoing bypass surgery. Ann Thorac Surg 2015;99:123-9.

26. Ando G, Morabito G, de Gregorio C, et al. Age, glomerular filtration rate, ejection fraction, and the AGEF score predict contrast-induced nephropathy in patients with acute myocardial infarction undergoing primary percutaneous coronary intervention. Catheter Cardiovasc Interv 2013;82:878-85.

27. Ando G, de Gregorio C, Morabito G, et al. Renal function-adjusted contrast volume redefines the baseline estimation of contrast-induced acute kidney injury risk in patients undergoing primary percutaneous coronary intervention. Circ Cardiovasc Interv 2014;7:465-72.

28. Wykrzykowska JJ, Garg S, Onuma Y, et al. Value of age, creatinine, and ejection fraction (ACEF score) in assessing risk in patients undergoing percutaneous coronary interventions in the 'All-Comers' LEADERS trial. Circ Cardiovasc Interv 2011:4:47-56.

29. Barili F, Pacini $D$, Rosato $F$, et al. In-hospital mortality risk assessment in elective and non-elective cardiac surgery: a comparison between EuroSCORE II and age, creatinine, ejection fraction score. Eur J Cardiothorac Surg 2014;46:44-8.

30. Yang $\mathrm{CH}$, Chang $\mathrm{CH}$, Chen $\mathrm{TH}$, et al. Combination of urinary biomarkers improves early detection of acute kidney injury in patients with heart failure. Circ J 2016;80:1017-23.

31. Chang $\mathrm{CH}$, Yang $\mathrm{CH}$, Yang $\mathrm{HY}$, et al. Urinary biomarkers improve the diagnosis of intrinsic acute kidney injury in coronary care units. Medicine (Baltimore) 2015;94:e1703. 